\title{
Erratum
}

\section{Unobstructed symplectic packing for tori and hyper-Kähler manifolds}

\author{
[J. Topol. Anal., Vol. 8, No. 4 (2016) 589-626] \\ Michael Entov \\ Department of Mathematics \\ Technion - Israel Institute of Technology \\ Haifa 32000, Israel \\ entov@math.technion.ac.il \\ Misha Verbitsky \\ Laboratory of Algebraic Geometry \\ National Research University HSE \\ Faculty of Mathematics \\ 7 Vavilova Str. Moscow, Russia \\ verbit@mccme.ru
}

Published 13 November 2018

In the proof of Theorem 9.2 in Section 9.2 of the paper [1] it was erroneously claimed without proof that the Teichmüller space of Kähler forms ${ }^{\mathrm{a}}$ on $T^{2 n}$ is connected (meaning that any two cohomologous Kähler forms on $T^{2 n}$ are isotopic as Kähler forms). While we do not know whether this claim is true, the proof of Theorem 9.2 in [1] in the case of $T^{2 n}$ (the only place in 1] where the claim was used) can be fixed in the following way (see 2] for more details).

Below we use the notations of 1 .

Consider the subgroup Diff ${ }_{H} \subset$ Diff $^{+}$, consisting of all diffeomorphisms of $T^{2 n}$ acting by identity on $H^{*}\left(T^{2 n}\right)$. The action of $\operatorname{Diff}_{H}$ on the Teichmüller space Teich of Kähler forms (that is, the space of the Kähler forms on $T^{2 n}$, factorized by the action of Diff 0 ) preserves the fibers of the period map Per : Teich $\rightarrow H^{2}(M, \mathbb{R})$ (that is, the map associating to an element of Teich ${ }_{s}$ the cohomology class of any Kähler form representing that element).

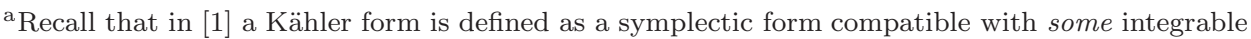
complex structure. 
We claim that this action is transitive on each fiber. ${ }^{\mathrm{b}}$

Indeed, by Proposition 6.1 of [1], any Kähler form on $T^{2 n}$ can be identified with a linear symplectic form by a diffeomorphism of $T^{2 n}$. Moreover, with an appropriate choice of the map $F$ appearing in the proof of that proposition, the latter diffeomorphism can be chosen to belong to Diff ${ }_{H}$. Since two linear cohomologous forms on $T^{2 n}=\mathbb{R}^{2 n} / \mathbb{Z}^{2 n}$ coincide, we get that any two cohomologous Kähler forms on $T^{2 n}$ — that is, representatives of two points of Teich ${ }_{s}$ lying in the same fiber of Per - can be mapped into each other by a diffeomorphism from Diff ${ }_{H}$, which proves the claim.

The transitivity of the action of Diff $_{H}$ on the fibers of Per and the fact that Per is a surjective local diffeomorphism imply that for any Kähler form $\omega$ on $T^{2 n}$ the orbit of the image of $\omega$ in Teich $s$ under the action of Diff ${ }^{+} / \mathrm{Diff}_{0}$ is dense in $\mathrm{Teich}_{s}$ if and only if the orbit of the cohomology class $[\omega]$ under the action of $\mathrm{Diff}^{+} / \mathrm{Diff}_{0}$ on $H^{2}\left(T^{2 n}, \mathbb{R}\right)$ is dense in the image of Per. The rest of the proof of Theorem 9.2 proceeds as in [1].

\section{References}

1. M. Entov and M. Verbitsky, Unobstructed symplectic packing for tori and hyper-Kähler manifolds, J. Topol. Analysis 8 (2016) 589-626.

2. M. Entov and M. Verbitsky, Unobstructed symplectic packing for tori and hyper-Kähler manifolds, arXiv:1412.7183v4 (2018).

3. A. E. Hatcher, Concordance spaces, higher simple homotopy theory, and applications, in Proc. Sympos. Pure Math., Vol. 32 (AMS, Providence, RI, 1978), pp. 3-21.

4. W. C. Hsiang and R. W. Sharpe, Parametrized surgery and isotopy, Pacific J. Math. 67 (1976) 401-459.

${ }^{\mathrm{b}}$ Let us note that for $T^{2 n}, n \geqslant 3$, the group Diff ${ }_{H} / \operatorname{Diff}_{0}$ is infinite (see [3 Thm. 4.1], [4, Thm. 2.5]). 